\title{
THE STUDY OF THE DISCOURSE RELATED TO THE CODE OF ROBOETHICS FOR THE HUMAN-ROBOT INTERACTION PROFESSION IN SUJOY GHOSH'S ANUKUL
}

\author{
Dr. Panchali Mukherjee \\ Associate Professor, Department of Arts and Humanities, Christ Academy Institute for Advanced Studies \\ Institutional Address: Christ Nagar, Hullahalli, Begur-Koppa Road, Sakkalawara Post, Bangalore 560083
}

\begin{abstract}
The research paper primarily studies the idea or discourse related to the code of "Robot Ethics" or "Roboethics" which governs human-robot interaction profession and is embodied in Sujoy Ghosh's film narrative Anukul (2017). It foregrounds the theory of "discursive formations" that propounds the formulation of knowledge from discourses that pre-exist the subject's experiences. The paper shows that the subject is not an autonomous or unified identity but is in process as a result there is a parallel shift in the history and philosophy of science. The paper attempts to explore the evolution of the "Robot Ethics" in the context of the film. It attempts to show that science progresses in discontinuous movement from one discursive formation or paradigm to another in connection to the development in the code of "Roboethics" as projected in the film narrative. The paper shows that the scientists conduct and write up their research within the conceptual limits of particular scientific discourses which are historically situated in relation to their society and culture. It shows that discourse related to "Robot Ethics" is connected to power. The research paper shows that individuals are subjects of ideology and the ideology/ies operate by the interpellation of the subjects in the social structure. This interpellation works through the discursive formations which are materially linked with "state apparatuses" such as religion, law and education.
\end{abstract}

Keywords: Roboethics, Human-Robot

Interaction, Discursive Formations

Introduction:

Discourse is speech or writing and the discourse that is embodied in Sujoy Ghosh's film Anukul (2017) is related to "Robot Ethics" also known as "Roboethics" which deal with the ethical concerns that result from using robots in various fields such as in healthcare or in war. Anukul is a story that refers to the humanoid robot Anukul who gets employment in the house of Nikunj Chaturvedi, a school teacher in Hindi, who has bought him from Chowringhee Robot Supply Corporation. When Nikunj Babu buys the robot, the salesperson warns him that no one should hit Anukul. He is meant to be employed as a domestic assistant in the house of Nikunj Babu. Unfortunately, Ratan, Nikunj Babu's cousin, who has been fired from his job visits Nikunj Babu's home and learns that the domestic assistant in his house is not a human being but a robot. This angers him as he lost his job because of such a robot and he hits Anukul with an iron. Anukul becomes unconscious and incurs damage. Nikunj Babu gets him repaired. After some time Ratan visits Nikunj Chaturvedi to give him the good news that he has become a millionaire. A relative of Ratan had died and all his wealth had been transferred to Ratan's name. As soon as Ratan goes out of Nikunj's eyesight Ratan tries to hit Nikunj as a result Anukul gives a high voltage electric shock to Nikunj because of which Ratan dies instantaneously. Later a lawyer visits Nikunj Babu and tells him that the wealth of Ratan that amounts to Rs. 11 and a half crores have been transferred to Nikunj Babu as Ratan has no descendants of his own. The film Anukul raises ethical concerns regarding the use of robots for various purposes. When the plot of the film is analyzed it makes the fact evident that robots are not supposed to harm human beings but they may do so in self-defense as done by Anukul to save himself from Ratan. This aspect brings to the mind the three "Laws of Robotics" as given by Isaac Asimov in 1940. They are the following:

First Law: A robot may not injure a human or through inaction, allow a human to come to harm. 
Second Law: A robot may obey the orders given it by human beings, unless such orders would conflict with the first law.

Third Law: A robot must protect its own existence, as long as such protection does not conflict with the first or second law.

Anukul violates the first law in self-defense thereby partially upholding the third law. Anukul's attempt to protect himself inadvertently leads to the death of Ratan although its unintentional. The high voltage electric shock was given to Ratan by Anukul in order to paralyze him but it leads to his death because at the same time there is lightning in the sky which electrocutes him. This incident is futuristic but results in the questioning of the ethical concerns operational in the domain of Robotics and a need is felt for the formulation of a code of ethics termed collectively as "Roboethics".

The ethical concerns are whether robots pose any threats to the human beings or not and whether the use of robots are problematic or not. The "Robot Ethics" have legal as well as socio-economic concerns. Since the robots are being transitioned into human social environments (HSEs) so a code of ethics has to be formulated which would govern the domain of "Human-Robot Interaction" (HRI) and its practitioners thereby designing the robots for such spaces. The guidance related to "Robot Ethics" is provided to HRI practitioners by institutional review boards or (IRBs). In the United States of America, the majority of consumer robots sold require little ethical oversight before they are sold. Thus, there is a need for a code of ethics for HRI practitioners not only in research roles but also in non-research roles ("A Code of Ethics for the Human-Robot Interaction Profession"). Anukul, the humanoid robot, in the film of the same name is shown to be a benign domestic assistant but when provoked by Ratan can prove to be fatal thereby elucidating the fact that robots may pose threats to the human beings and their use can be problematic in certain cases. Thus, raising legal and socio-economic concerns. The fact that robots are being transitioned into human social environments (HSEs) is evident when Nikunj Chaturvedi buys Anukul from Chowringhee Robot Supply Corporation to be a domestic assistant in his household. Anukul has a seamless resemblance with a human being and the sales representative at Chowringhee Robot Supply Corporation tells Nikunj Chaturvedi that Anukul has been designed in such a way that he can do all kinds of work. Nikunj
Chaturvedi is told by the salesperson that Anukul should not be hit by anyone whatsoever thereby providing guidance to Nikunj Babu regarding HRI. Anukul is expected to engage in "Human-Robot Interaction" as he will not only interact with Nikunj Babu in a non-research role but also with other human beings. Thus, the need is felt for a code of ethics in relation to HRI.

The theory of "discursive formations" refuses to separate subject and object into separate domains. Knowledges are always formed from discourses which pre-exist the subject's experiences. Even the subject itself is not an autonomous or unified identity but is always in process. T.S. Kuhn and Paul Feyerabend have challenged the belief in the steady progression of knowledge in the sciences which is in a discontinuous movement from one discursive formation or paradigm to another. Discourse determines the criteria of truth based on authority and is connected to power. Thus, the scientists conduct and write up their research within the conceptual limits of particular scientific discourses which are historically situated in relation to their society and culture. There are distinctive ethical challenges which have arisen in HRI research, development and marketing. A need for ethics programming has been felt in artificial systems. This shows the progression of knowledge in the domain of science leading to a discontinuous movement from one discursive formation to the other. The robots are considered to be embodied systems capable of directly enacting physical change in the world. The ethical issues are applicable to all robots in HSEs regardless of their level of autonomy, their role, their capabilities or their morphology ("A Code of Ethics for the Human-Robot Interaction Profession"). The creation of the robots initially in research roles and then in non-research roles such as Anukul in the film of the same name is the brainchild of the scientists working on robots and is shown to be in a non-research role that of a domestic assistant. The diversification of the robots into domestic sphere as well as into other professional spheres in non-research roles is a result of the development in the knowledge of the sciences which has come about due to the advancement in science and technology. This advancement has been brought about by the evolution of the scientific minds which are always in process. The diversification of the robots is need based depending on the needs of the human beings. 
The film shows that initially the robots that were deployed especially in research roles in a laboratory set-up with the passage of time have been transferred in non-research roles to human social environments and their acceptance in the human domain is considered to be a significant development of the recent times thereby showing the discontinuous movement from one discursive formation to the other. The film highlights that the subjects in this case may be considered as the scientists who at a point in time envisioned the creation of robots who would serve in research roles in a laboratory set-up but due to advancement in "Artificial Intelligence" and a need being felt by the same scientists for robots in non-research roles in human social set-ups. Since discourse determines the criteria of truth based on authority and is connected to power the diversification of robots is an idea that is acceptable for currently emerging situations and futuristic situations. Thus, the scientists have written up their research within the conceptual limits of particular scientific discourses such as robotics in this case, which are historically situated in relation to their society and culture as modern times ushered in the rise of "Artificial Intelligence" to cater to the needs of the human population and in turn simplify their lives.

The robots are of various types namely therapeutic robots, physically assistive robots and robot interrogators. The therapeutic robots are deployed in therapeutic settings with vulnerable populations. The physically assistive robots provide physical assistance to people with disabilities. Since the social robots are marketed and the human-robot interaction becomes more frequent across a wider array of settings with robots functioning as sales agents, conflict resolution intermediaries etcetera the common role is that of the robot interrogator. HRI practitioners often use Wizard-of-Oz (WoZ), a technique where a person remotely operates a robot and puppeteers many of its attributes such as speech, non-verbal behavior, navigation, manipulation etcetera. WoZ involves multiple degrees of control as well as mixed initiative interaction ("A Code of Ethics for the Human-Robot Interaction Profession"). Anukul too is programmed and is remotely operated in terms of speech, non-verbal behavior, navigation, manipulation etcetera.

The therapy recipients of the therapeutic robots may develop strong psychological and emotional bonds with them the severing of which at the end of a project can have serious harmful effects on the concerned subjects thereby negating any therapeutic benefit the subject might have experienced or even leaving the subject in worse condition than before the research began. Therefore, the HRI research needs to address the risks and benefits for the human subjects associated with the termination of the research program with protocols being specified in advance for addressing any consequent needs on the part of the subject. The design of physically assistive robots must take into consideration the privacy rights of clients such as the deactivation of video monitors during intimate procedures. Care should be taken with protocols for touching. The HRI practitioners should consider whether robots should be designed to encourage and discourage the formation of emotional bonds while realizing some bonding will be inevitable regardless of the morphology of the platform. The interaction of robot interrogators with human customers involve unintended information disclosure which can be used for commercial purposes. The burden for managing risk shifts is ever more from the human to the HRI practitioners. There are ethical concerns raised by researchers about $\mathrm{WoZ}$ and its inherent social deception. The researchers discuss the problems with "Turing Deceptions" where a participant cannot determine if they are interacting with a machine, a specific person or a person masquerading as another person. The users should be able to realize on a WoZ interaction because robotic intelligence remains an illusion. An issue to consider in relation to $\mathrm{WoZ}$ is the problem of fostering inappropriate expectations among people interacting with the robot ("A Code of Ethics for the Human-Robot Interaction Profession"). The film exhibits two instances one of emotional bonding and the other of emotional dissociation. Nikunj Chaturvedi develops an emotional bonding with Anukul, a humanoid robot or an android, over a period of time as he is a domestic assistant in the household of the otherwise lonely Nikunj Babu and is instrumental in him gaining wealth. On the other hand, Ratan, Nikunj Chaturvedi's cousin, who has lost his job for such a robot is emotionally dissociated from Anukul so much so that he hits Anukul once thereby making him unconscious.

There is lack of diversity in robot morphology and behavior especially regarding manifestations of gender, race and ethnicity. Another issue concerning diversity of platforms is 
that many mechanistic robots conform to Hollywood-driven stereotypes ("A Code of Ethics for the Human-Robot Interaction Profession"). In this case, Anukul's morphology and behavior is that of a human being who is extremely sober, wellmannered, soft-spoken and industrious.

The individuals are subjects of ideology and the ideology/ies operate by the interpellation of the subjects in the social structure. This interpellation works through the discursive formations which are materially linked with "state apparatuses" such as religion, law and education. Thus, the HRI code of ethics refers to the following principles under these considerations namely:

\section{Human Dignity Considerations:}

The emotional needs of humans are always to be respected. The humans' right to privacy shall always be respected to the greatest extent consistent with reasonable design objectives. Human frailty both physical and psychological is always to be respected ("A Code of Ethics for the Human-Robot Interaction Profession"). In Anukul, Anukul tolerates the anger of Ratan, the cousin of Nikunj Babu, as he knows that humans are susceptible to anger and in Ratan's case he is angry because he lost his job to a robot thereby respecting the emotional needs of humans. Nikunj Chaturvedi, Anukul's employer, forms an emotional attachment towards him as a result of the facts that Anukul not only provides companionship to Nikunj Chaturvedi who lives alone moreover, Anukul is instrumental in Nikunj Babu getting a legacy of Rs. 11 and a half crores which comes about when Anukul gives a high voltage shock to Ratan, Nikunj Babu's cousin, and his wealth passes on to Nikunj Babu.

\section{Design Considerations:}

Maximal, reasonable transparency in the programming of robotic systems is required. Predictability in robotic behavior is desirable. Trustworthy system design principles are required across all aspects of a robot's operation for hardware and software design as well as data processing on or off the platform. Real-time status indicators should be provided to users to the greatest extent consistent with reasonable design objectives. Obvious opt-out mechanisms (kill switches) are required to the greatest extent consistent with reasonable design objectives ("A Code of Ethics for the Human-Robot Interaction Profession"). In Anukul, Anukul the android when bought by Nikunj Chaturvedi from Chowringhee Robot Supply Corporation Nikunj
Babu is instructed regarding the use of such androids. It is told to him by the salesperson that Anukul is capable of doing any kind of work, he does not sleep and he should not be hit by anyone. These guidelines are provided to Nikunj Babu by the salesperson at the time of buying Anukul since Nikunj Babu is the customer or the end user who would use Anukul.

\section{Legal Considerations:}

All relevant laws and regulations concerning individuals' rights and protections are to be respected. A robot's decision paths must be reconstructible for the purposes of litigation and dispute resolution. Human informed consent to HRI is to be facilitated to the greatest extent possible consistent with reasonable design objectives ("A Code of Ethics for the Human-Robot Interaction Profession"). In the film, the death of Ratan is more of an accident and the high voltage electric shock as given by Anukul to Ratan is in self-defense but the legal implication related to it is not taken into consideration in the ambit of the film except for the fact that the wealth that Ratan had inherited from a dead uncle of his was passed on to Nikunj Babu after Ratan's accidental death.

\section{Social Considerations:}

Wizard-of-Oz should be employed as judiciously and carefully as possible and should aim to avoid Turing deceptions. The tendency for humans to form attachments to and anthropomorphize robots should be carefully considered during design. Humanoid morphology and functionality are permitted only to the extent necessary for the achievement of reasonable design objectives. Avoid racist, sexist and ableist morphologies and behaviors in robot design ("A Code of Ethics for the Human-Robot Interaction Profession"). In Anukul, Anukul is a Wizard-of-Oz which is pre-programmed although the Turing deceptions that result from them are unintentional but inevitable as his morphological and functional characteristics are similar to that of a human being. Ratan, Nikunj Chaturvedi's cousin, is shown to have no emotional attachment towards Anukul and in fact harbors militant thoughts in relation to robots as he lost his job because of such a robot coming into existence. As a result, Ratan tries to harm Anukul by hitting him with an iron because of which Anukul becomes unconscious and incurs damage. Anukul, the android or the humanoid robot is shown to be anthropomorphized in the short film of the same name as its morphology is that of an 
Indian Hindi speaking male and his functionality is that of a domestic assistant although he is a robot. $\mathrm{He}$ is anthropomorphized as he has to be integrated in the household of Nikunj Babu who is a human being and serve the purpose of a domestic assistant so he should seem like a human being. The racist, sexist and ableist morphologies and behaviors have been avoided in the case of the robot design of Anukul as he appears as an Indian and works among Indians who are males, he does not display aggressive behavior and is not shown to possess any morphological disabilities.

A viable code of "Roboethics" is required for the effective functioning of the robots as in futuristic scenarios more and more robots will be developed in non-research roles in addition to research roles and they will be functional in various domains as shown in the film Anukul. This will give rise to innumerable situations related to HumanRobot Interaction (HRI) which may have to be monitored or supervised by applying the code of "Roboethics" and will result in dealing with the human dignity, design, legal and social considerations operational in the domain. The code is primarily based on Isaac Asimov's "Three Laws of Robotics". The formulation of "Roboethics" is in flux as this field is subjected to continuous advancement or progression. The film which is based on Satyajit Ray's short story "Anukul" shows the challenges that the androids may have to face when they are incorporated in various spheres in non-research roles and the threats that human beings face from them in the form of unemployment problem as shown in the film leading to an aversion towards "Artificial Intelligence". The robots, although the creation of human beings, are smarter in terms of intelligence and have greater adaptability compared to humans so much so that they are being developed in various roles to cater to the needs of the human beings. Anukul is a prime example of such kind of a robot which is smart and has high adaptability and is fit for all kinds of tasks. Anukul is also shown to broaden the horizon of his knowledge in the film by reading Bhagvad Gita and trying to understand the religious concepts of Hinduism which in fact elucidates his skills related to adaptability and he having higher form of intelligence. The film shows Anukul's seamless integration into the human world and he is a Wizardof-Oz with complete "Turing Deceptions" as in the course of the film Ratan is initially unable to understand whether Anukul is a robot or a human being. The need for a code of "Roboethics" is felt by the scientists all the more as they can envision that in futuristic scenarios there will be a rapid increase in Human-Robot Interaction resulting in creation of robots which have the ability to outwit the human brain. Thus, the discourses related to formulation and further advancement in "Roboethics" have emerged as a result of the scope that such discourses have in an age that is considered to be significant in the development of "Artificial Intelligence" and may prove to be the launch pad for a more evolved code of "Roboethics" for futuristic scenarios.

\section{Works Cited}

1. Anderson, M. and Anderson, S.L., ed. Machine Ethics. Cambridge: Cambridge University Press. 2011.

2. Arkin, R.C. Governing Lethal Behavior in Autonomous Robots. New York" Taylor and Francis Group, 2009.

3. Draude, C. "Intermediaries: Reflections on Virtual Humans, Gender, and the Uncanny Valley." AI \& Society 26.4 (2011): 319327.

4. Form, W. "On the Degradation of Skills." Annual Review of Sociology (1987): 29-47.

5. Ghosh, Sujoy, dir. Anukul. 2017. 1 Jan. 2019

6.

https://www.youtube.com/watch?v=J2mqI gdae 5I

7. Goodrich, M. A. and A.C. Schultz. "Human-Robot Interaction: A Survey." Foundations and Trends in HumanComputer Interaction 1.3 (2007): 203-275.

8. Kulić, D. and E. A. Croft. "Safe Planning for Human-Robot Interaction." Journal of Robotic Systems 22.7 (2005): 383-396.

9. Miller, K.W. "It's Not Nice to Fool Humans.” IT Professional 12.1 (2010).

10. Nourbakhsh, I. R. Robot Futures. Amherst: MIT Press, 2013.

11. Riek, L.D. "Wizard-of-Oz Studies in HRI: A Systematic Review and New Reporting Guidelines." Journal of Human Robot Interaction 1.1 (2012). 
12. ........... and Don Howard. "The Code of Ethics for the Human-Robot Interaction Profession." We Robot. 2014.1 Jan. 2019

13. http://robots.law.miami.edu/2014/wpcontent/uploads/2014/03/a-code-of-ethicsfor-the-human-robot-interaction-

profession-riek-howard.pdf 